Sub-grid effects of the Voigt viscoelastic regularization of a singular dyadic model of turbulence

This content has been downloaded from IOPscience. Please scroll down to see the full text.

2013 J. Phys. A: Math. Theor. 46195501

(http://iopscience.iop.org/1751-8121/46/19/195501)

View the table of contents for this issue, or go to the journal homepage for more

Download details:

IP Address: 146.164.6.222

This content was downloaded on 02/05/2017 at 21:27

Please note that terms and conditions apply.

You may also be interested in:

Finite size Lyapunov exponent: review on applications

Massimo Cencini and Angelo Vulpiani

Continuous representation for shell models of turbulence

Alexei A Mailybaev

Universal properties of three-dimensional magnetohydrodynamic turbulence: do Alfv'enwaves matter?

Abhik Basu and Jayanta K Bhattacharjee

Statistics of the Navier--Stokes-alpha-beta regularization model for fluid turbulence

Denis F Hinz, Tae-Yeon Kim and Eliot Fried

Quasi-two-dimensional turbulence

Sergei D Danilov and D Gurarie

Astrophysical turbulence modeling

Axel Brandenburg and Åke Nordlund

Simulations of Supersonic Turbulence

Alexei G. Kritsuk, Michael L. Norman, Paolo Padoan et al.

EQUILIBRIA, DYNAMICS AND CURRENT SHEETS FORMATION IN MAGNETICALLY CONFINED CORONAE

A. F. Rappazzo

Progress in studying small-scale turbulence using 'exact' two-point equations

L Danaila, R A Antonia and P Burattini 


\title{
Sub-grid effects of the Voigt viscoelastic regularization of a singular dyadic model of turbulence
}

\author{
Rafael Borges and Fabio Ramos \\ Departamento de Matemática Aplicada, Universidade Federal do Rio de Janeiro, Rio de Janeiro, \\ RJ 21945-970, Brazil \\ E-mail: framos@ufrj.br
}

Received 17 December 2012, in final form 2 April 2013

Published 29 April 2013

Online at stacks.iop.org/JPhysA/46/195501

\begin{abstract}
In this work we investigate the spectral signature of Navier-Stokes-Voigt (NSV) viscoelastic fluid flows by employing numerical simulations of a singular dyadic shell model. Our results clearly show that as the relaxation time is increased above a threshold, the inertial range is reduced, conserving part of the large-scale statistics. These results differ drastically from the two power-law scenarios observed in a previous work, where the NSV model was studied via Sabra shell model simulations instead. We also show that the additional elastic term regularizes the singular dyadic model, which is the main reason behind this reduction of degrees of freedom. The results of this work aim at proposing the NSV regularization as a sub-grid model.
\end{abstract}

PACS numbers: 47.35. $-\mathrm{i}$, 47.27.- $\mathrm{i}$, 83.60.Df, 47.10.ad, 02.40.Xx

Mathematics Subject Classification: 35Q30, 35Q35, 76F20, 76F55

(Some figures may appear in colour only in the online journal)

\section{Introduction}

In spite of being the subject of intensive research for several decades, many details of the statistical theory of turbulent flows remain elusive. One of the cornerstones of the theory, developed by Kolmogorov in his groundbreaking works of the early 1940s, predicts that in a certain range of length scales, where neither dissipation nor energy input are dominant, the $n$ th-order structure function of the velocity field of turbulent flows should obey the scaling law $S_{n}(r) \sim C \epsilon^{n / 3} r^{n / 3}$, where $\epsilon$ is the mean energy dissipation rate and $C$ is a universal constant. However, experiments show that this law can be considered correct only for the moments of orders 2 and 3, but incorrect for higher order. The true nature and ultimate causes of this intermittent behavior are still subjects of great scientific disputes to this day; see [16] for details. 
Large fluctuations of small-scale structures, mainly due to boundary effects, may be the source of this deviation, as was first noticed by Landau, who challenged the universality assumption, see [16, section 6.4]. It is important to remark that most of Kolmogorov's predictions in his landmark papers were based on purely dimensional arguments, i.e. the Navier-Stokes (NS) equations played a very small role.

Different features of Kolmogorov theory were independently discovered by many other scientists, including Lars Onsager, who after rederiving the Kárman-Howarth relation and the Kolmogorov 4/5th law, proposed that weak solutions of Euler equations could develop singularities in finite time, which can be the ultimate source for the power-law scaling of the structure functions; see $[11,12]$ for more details. This is similar to the phenomenology of Burgers' equation, see $[7,16]$.

Debates about the universality assumption, the correct scaling of the structure functions and the role played by singularities remain central topics in the fluid mechanics community.

Another consequence of K41 theory is the existence of a dissipative range of scales, below the Kolmogorov dissipative length scale, where the effects of viscosity are dominant. This range, despite carrying only a small fraction of the total kinetic energy of the flow, represents the vast majority of scales involved in direct numerical simulations; see [30, 39].

The main objective of sub-grid models is to accurately reproduce the large-scale details of turbulent flows without the need to compute too much of the dissipative range, which is the main source of computational costs. This can be achieved in many ways, but they essentially involve modeling the energy transfer between large and small scales through the inertial nonlinear term; see [30, 39] for more details.

Understanding the intricate details of the interplay between the inertial range and the dissipation range is crucial not only for numerical analysis purposes, but also for understanding the still very mysterious physics of viscoelastic flows; see [33]. For example, the phenomenon of drag reduction in viscoelastic fluids is one of the most studied topics in the fluid mechanics community, and even after many years of scientific efforts, most of the details behind this phenomenon are still elusive; see [35, 43, 47]. Study [43] is one which is phenomenologically more connected to ours, since it proposes an explanation for the phenomena based on the suppression of the energy cascade due to elastic interactions.

Recently, a related phenomenon still very poorly understood has attracted much attention from the turbulence community. It is the elastic turbulence phenomenon arising from viscoelastic flows, which displays many features of turbulence even for very small Reynolds numbers; see [42] and references therein. This may have important applications for heat transfer problems in micro-channels.

The complex behavior of this kind of flow is mainly due to the nonlinear interaction of its inner polymeric structures and the solvent, and it leads to very complex and ingenuous micro-macro models (see [10]) which are often very difficult to analyze either analytically or numerically.

A long-time tradition of analyzing viscoelastic materials from a purely macroscopic point of view comes from the days of Kelvin, Maxwell and Voigt; see [45, 46, 48]. It amounts to the consideration of appropriate modifications of the Cauchy stress tensor, which ultimately yields its viscoelastic nature. This way of modeling complex viscoelastic flows is certainly very naive, but it does play an important role in grasping some of the fundamentals in this very complex setting.

In this work, we give continuity to the study presented in [28], where it was proposed that a viscoelastic macroscopic model, the Navier-Stokes-Voigt (NSV) equations, could be used as a sub-grid model. Indeed, it is our belief that a large family of viscoelastic models, which are based on timescale modifications, can be used as sub-grid models. 
One of the main advantages of the NSV model compared to other regularization and sub-grid scale models, such as hyperviscosity or $\alpha$-models, is the fact that it does not require any artificial boundary conditions, which cause difficulties and possibly exhibit non-physical behavior in applications, such as non-physical boundary layer; see, e.g., [14].

In [28], the spectral behavior of the NSV equations was investigated via shell model simulations. Let us briefly comment on the NSV model. In the purely macroscopic approach, the main rheological properties of a viscoelastic flow are defined by its constitutive law and, in particular, by its Cauchy stress tensor. For Kelvin-Voigt materials, it is given by

$$
\sigma=-p \mathbf{I}+2 \mu \mathbf{D}+2 \beta \frac{\partial \mathbf{D}}{\partial t}
$$

where $p \mathbf{I}$ is the normal stress, related to the incompressibility condition, $\mathbf{D}$ is the symmetric component of the velocity gradient and $\frac{\partial \mathbf{D}}{\partial t}$ is the stretch tensor. The coefficient $\mu$ is the shear viscosity and $\beta$ is the shear modulus of the elastic component.

Considering this class of stress tensor for incompressible fluids, and substituting it into the linear momentum balance, gives rise to the equations of motion for the NSV fluids:

$$
\left\{\begin{array}{l}
\partial_{t}\left(\mathbf{u}-\alpha^{2} \Delta \mathbf{u}\right)-v \Delta \mathbf{u}+\mathbf{u} \cdot \nabla \mathbf{u}+\nabla p=\mathbf{f}, \quad \mathbf{x} \in \Omega, \\
\nabla \cdot \mathbf{u}=0, \quad \mathbf{x} \in \Omega, \\
\mathbf{u}(\mathbf{x}, 0)=\mathbf{u}_{0}(\mathbf{x}), \quad \mathbf{x} \in \Omega, \\
\mathbf{u}(\mathbf{x}, t)=0 \quad \mathbf{x} \in \partial \Omega, \quad \text { or } \quad \mathbf{u}(\mathbf{x}, t) \text { is periodic; }
\end{array}\right.
$$

in the domain $\Omega\left(\Omega=[0, L]^{3}\right.$ in the periodic case), where $\alpha \geqslant 0$ is a given length-scale parameter and $v>0$ is a given kinematic viscosity, such that $\alpha^{2} / v$ is the relaxation time of the viscoelastic fluid. Of course, if we set $\alpha=0$, we recover the incompressible NS equations.

We will discuss some of the properties of the NSV equations in the following section, but we would now like to point out the recent work [27] in which the authors test the Voigt regularization in benchmark problems of the two-dimensional NS and magnetohydrodynamic (MHD) equations. Their results show the efficacy of this regularization in numerical simulations performed in a coarse grid in domains with physical boundaries, building up more evidence that this model can be used as a sub-grid model.

Numerical simulations of the full three-dimensional NSV equations, however, are certainly an extremely demanding task for very high Reynolds number flows, just like for the NS equations.

In order to get an insight into the turbulence problem, several simplified models have been developed in the past. One of the most studied models is the shell model of turbulence, which is a phenomenological model inspired by a severe truncation and averaging of the equations of motion in Fourier space. Shell models of turbulence have a long history in the fluid mechanics community since the pioneering works of Obukhov; see [16]. These models possess conserved quantities compatible with the unforced 3D incompressible Euler equations, and simulations are usually performed with forcing concentrated in the low modes, with a quadratic viscous dissipation inspired by the NS equations. The main weakness of these models is the absence of geometrical information in the real space, but, nevertheless, it is very useful to test simple ideas concerning the statistical spectral dynamics of turbulent flows.

In [28], the statistical properties of the Voigt regularization have been studied by the second author and collaborators, where the effects of the Voigt regularization to the Sabra shell model of turbulence were described. The equations of motion of the Sabra shell model of turbulence were introduced in [36], and they have the following form:

$\frac{\mathrm{d} u_{n}}{\mathrm{~d} t}=\mathrm{i}\left(a k_{n+1} u_{n+2} u_{n+1}^{*}+b k_{n} u_{n+1} u_{n-1}^{*}-c k_{n-1} u_{n-1} u_{n-2}\right)-v k_{n}^{2} u_{n}+f_{n}$ 


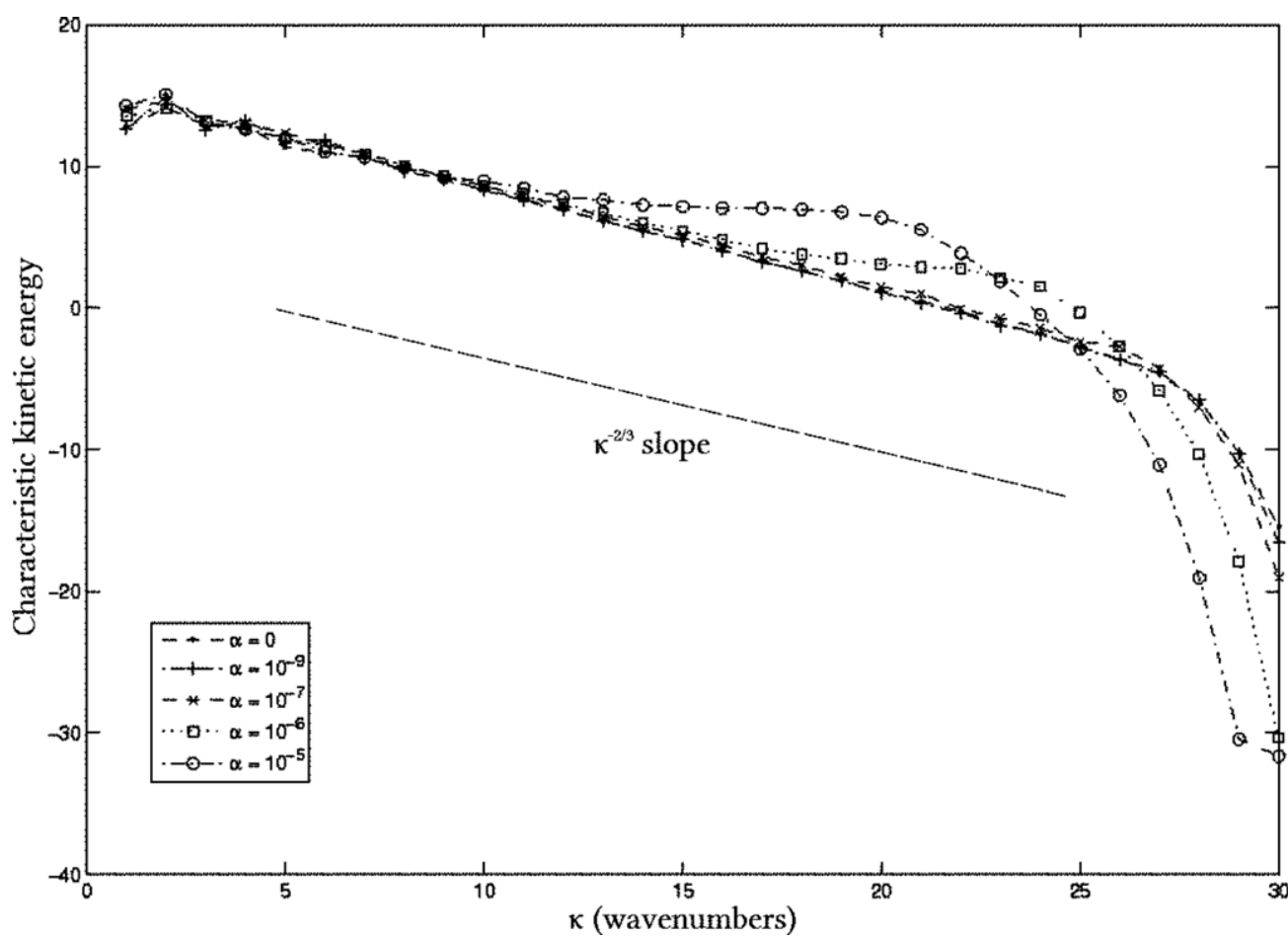

Figure 1. $\log -\log$ plot of the $S_{2}$ structure function of the Sabra-Voigt model with viscosity = $v=10^{-12}$ and several values of $\alpha$. Forcing: constant on the third and fifth modes. This figure was taken and adapted from [28].

for $n=1,2,3, \ldots$, with the boundary conditions $u_{1}=u_{0}=0$. The wavenumbers $k_{n}$ are taken as $k_{n}=\lambda^{n}$, with $\lambda>1$ being the shell spacing parameter. Although the equation does not capture any geometry, the scale $L=k_{0}^{-1}$ is frequently considered as a fixed typical length scale of the model. In an analogy to the NS equations, $v>0$ represents a kinematic viscosity and $f_{n}$ are the Fourier components of forcing.

The Voigt-Sabra equations are a simple adaptation of the Sabra model described above. The equations are

$$
\left(1+\alpha^{2} k_{n}^{2}\right) \frac{\mathrm{d} u_{n}}{\mathrm{~d} t}=\mathrm{i}\left(a k_{n+1} u_{n+2} u_{n+1}^{*}+b k_{n} u_{n+1} u_{n-1}^{*}-c k_{n-1} u_{n-1} u_{n-2}\right)-v k_{n}^{2} u_{n}+f_{n},
$$

where, as in (1), $\alpha \geqslant 0$ is a given length-scale parameter and $v>0$ is a given kinematic viscosity, such that $\alpha^{2} / v$ is the relaxation time of the viscoelastic fluid. All the other parameters are as in (2).

In [28], two different scalings for the inertial range (see figure 1) and a relevant decrease of dissipation range intermittency were observed. It was argued that the regularization of possible singularities in the inviscid Sabra model may be the main reason for this behavior. But since the question of blow-up for the inviscid Sabra model has not yet been answered, this is really only a conjecture; see [9] for more details concerning the regularity of the Sabra model.

A large set of phenomenological assumptions in turbulence lead naturally to a large set of shell model types. Recently, several works analyzed the so-called dyadic model, which is known to produce both power-law scaling and Onsager-like singularities when viscosity is set 
to zero; see [3-5, 23]. The viscous dyadic model is the following system of nonlinear ordinary differential equations (ODEs):

$$
\frac{\mathrm{d} u_{n}}{\mathrm{~d} t}+v \lambda^{2 n} u_{n}-\lambda^{n} u_{n-1}^{2}+\lambda^{n+1} u_{n} u_{n+1}=f_{n}
$$

for $n=1,2,3, \ldots$, with the boundary condition $u_{0}=0$, and where $\lambda>1$ is the shell spacing parameter. As in (2), the wavenumbers $k_{n}$ are taken as $k_{n}=\lambda^{n}$, with $\lambda>1$ being the shell spacing parameter. In this work, we set $\lambda=2^{5 / 2}$.

In $[3,23]$, one can find a derivation of the dyadic model based on a Littlewood-Paley decomposition of the Euler equations, where each ODE represents a wavelet coefficient describing the behavior of the velocity localized to a certain frequency shell.

The inviscid dyadic model $(v=0)$ is known to blow-up in the $H^{5 / 6}$ norm, and to possess a global attractor in $L^{2}$, for certain choices of forcing. These global attractors do not belong to the space $H^{5 / 6}$, and they have a spectral scaling compatible with Onsager's theory. The mechanism of dissipation is provided by the singularity, similar to the Burgers' equations; see $[3-5,23]$. The main reason for this dissipation is the monotonic transfer of energy to high modes provided by the nonlinear term.

It has also been proved that when viscosity is positive, solutions remain regular and possess global attractors. These attractors converge to the singular global attractor as $v$ goes to zero, see [21, 29, 40].

In this work, we continue the investigation initiated at [28] by studying, analytically and numerically, the effects of the Voigt viscoelastic parameter on the dyadic model. The Voigt-dyadic system of equations is

$$
\left(1+\alpha^{2} \lambda^{2 n}\right) \frac{\mathrm{d} u_{n}}{\mathrm{~d} t}+v \lambda^{2 n} u_{n}-\lambda^{n} u_{n-1}^{2}+\lambda^{n+1} u_{n} u_{n+1}=f_{n}
$$

for $n=1,2,3, \ldots$, with the boundary condition $u_{0}=0$, and where $\lambda=2^{5 / 2}$ is the shell spacing parameter. The parameter $\alpha \geqslant 0$ plays the same role as described in (3). Again, here the wavenumbers $k_{n}$ are taken as $k_{n}=\lambda^{n}$.

We show that the Voigt term does regularize the singular inviscid dyadic model, and that dissipation range oscillations are indeed tamed by the additional regularization, with a significant reduction of the inertial range's size. We also show that Kolmogorov power-law scaling persists for viscous flows for any of the tested values of $\alpha$, without a secondary inertial range, which is in dissonance with our previous work. These results, if corroborated by more extensive and realistic DNS, imply that the NSV regularization may become a useful sub-grid model.

In section 2, we discuss the energy distribution scale by scale following the Kraichnan arguments involving conserved quantities and dimensional analysis. In conjunction with the numerical results, we argue for a transfer timescale which is different from the one observed in [28]. This shows the limitation of using shell models for investigating the correct scaling of new models.

We also discuss the issue of smallest scales of motion. Our numerical results clearly show a reduced inertial range, with no modification of the statistics of large scales. This is the main result of this paper, and this is the reason we argue for the NSV to be tested as a possible sub-grid model. We also present several relations between the dissipative cut-off scale and the parameters $\alpha$ and $v$.

In section 3, we offer our conclusions, and in the appendix we sketch the proof of the regularity of the Voigt-dyadic model. 


\section{The spectral distribution}

The NSV model of viscoelastic incompressible fluid has been studied by Oskolkov in $[32,37,38]$, where he proved its solvability in different functional spaces. The NSV model was further studied in $[2,29]$ where other regularity issues were proved. We will not discuss much about regularity issues for the NSV equations in this work, but we remark that these equations behave like a damped hyperbolic system; see [21]. Therefore, solutions do not experience fast (instantaneous) smoothening of the initial data, as is the case for parabolic systems like the NS equations.

However, it was proved in [21] that solutions in the global attractor are smooth if the forcing field is smooth enough, even for initial data satisfying only finite kinetic energy and finite enstrophy (i.e. bounded in the Sobolev $H^{1}$-norm). In particular, in [21] it was shown in the periodic case that if the forcing field is analytic, then the global attractor consists of analytic functions. This result, in conjunction with the results proved in [40], proves that if the forcing field is smooth enough, then averaged structure functions, with respect to an invariant measure for the NSV flow, display an exponentially decaying tail.

In this work, we show that this smoothening character of the NSV global attractor is not lost even for singular models such as the dyadic model.

The NSV model satisfies the following energy equation for every $t \in[0, \infty)$ :

$$
\frac{\mathrm{d}}{\mathrm{d} t}\left(\frac{1}{2}|\mathbf{u}(\cdot, t)|_{L^{2}}^{2}+\frac{\alpha^{2}}{2}|\nabla \mathbf{u}(\cdot, t)|_{L^{2}}^{2}\right)=(\mathbf{f}, \mathbf{u}(\cdot, t))_{L^{2}}-v|\nabla \mathbf{u}(\cdot, t)|_{L^{2}}^{2} .
$$

Therefore, a positive quadratic conserved quantity in the inviscid, $v=0$, unforced, $\mathbf{f}=0$, and periodic or no-slip setting, which was proved rigorously in [2], is

$$
S_{2}^{\alpha}=\frac{1}{2}|\mathbf{u}|_{L^{2}}^{2}+\frac{\alpha^{2}}{2}|\nabla \mathbf{u}|_{L^{2}}^{2}
$$

which we call the $\alpha$-energy. The quantity

$$
S_{2}=\frac{1}{2}|\mathbf{u}|_{L^{2}}^{2}
$$

is the usual kinetic energy, and we remark that it is not conserved for the inviscid unforced NSV equations.

Another conserved quadratic quantity is the $\alpha$-helicity:

$$
\Lambda_{\alpha}=\left(\mathbf{u}-\alpha^{2} \Delta \mathbf{u}, \operatorname{curl}(\mathbf{u})\right)_{L^{2}} .
$$

In order to study the statistical equilibrium spectral profile, we will employ the methods used in [25] (see also [13]). However, instead of considering the kinetic energy, we will analyze the ideally conserved $\alpha$-energy, $S_{2}^{\alpha}$, and conclusions will be further recovered for the kinetic energy, $S_{2}$. This method was also used in $[1,2,6,14,17,31,34,28]$ for studying various $\alpha$ regularizations of the NS equations.

The main difference of our work in comparison with those cited above is that we will consider the $\alpha$ regularization of a singular shell model, i.e. for which the kinetic energy is not ideally conserved when $\alpha=0$.

This singularity is associated with a very fast, and monotonic, energy transfer rate of the input energy from low wavenumbers to high wavenumbers. We show that the dimensional analysis argument carried out in [28] does not lead to the observed spectra in our numerical simulations. This shows the limitation of investigating $\alpha$-models via shell model simulations, since two different shell models may lead to different scaling for the same $\alpha$-model. 


\subsection{Singularity scaling}

The main mechanism behind the power-law scaling in the viscous dyadic model is the scaling of the singular global attractor for the inviscid dyadic model. The singular scaling persists for low wavenumbers because the viscous regularization acts mostly at high wavenumbers, leaving most of the large-scale statistics untouched.

To describe more precisely this singularity mechanism, we must now briefly introduce the relevant functional setting of this work. We first rewrite the NSV equations in a functional form and mimic the same procedure to the Voigt-dyadic model (keeping the same notation). Let $A$ be the Stokes operator defined by $A \mathbf{u}=-P_{\mathrm{LH}} \Delta \mathbf{u}$, where $P_{\mathrm{LH}}$ is the (Leray-Helmhotz) orthogonal projector over the divergence-free vector fields in $L^{2}(\Omega)^{3}$; see, e.g., $[8,15,44]$ for more details. For the sake of simplicity, we will consider only divergence-free forcing fields, i.e. $\mathbf{f}=P_{\mathrm{LH}} \mathbf{f}$. The term $B(\mathbf{u}, \mathbf{v})=P_{\mathrm{LH}}((\mathbf{u} \cdot \nabla) \mathbf{v})$ is a bilinear form associated with the inertial term. Let $\mathbf{u}$ and $\mathbf{v}$ be the divergence-free smooth functions in $\Omega$. The important orthogonality property holds (see, e.g., [8])

$$
(B(\mathbf{u}, \mathbf{v}), \mathbf{v})_{L^{2}}=0 .
$$

This relation holds only for sufficiently smooth velocity fields, and failure to fulfil the equivalent to this identity is the ultimate source of the anomalous dissipation in the singular dyadic model. The NSV model can be written in the functional form as

$$
\left(I+\alpha^{2} A\right) \frac{\mathrm{d} u}{\mathrm{~d} t}+v A u+B(u, u)=f .
$$

Let us now turn to the Voigt-dyadic model. We first define the relevant spaces and operators. Let $H=\ell^{2}$ be defined with the usual scalar product and norm

$$
(u, v):=\sum_{n=1}^{\infty} u_{n} v_{n}, \quad|u|:=\sqrt{(u, u)} .
$$

The $\alpha$-dyadic model can be written as in (11) in terms of the dyadic nonlinear operator $(B(u, u))$ and the dyadic Stokes operator $A: D(A) \rightarrow H$, defined in this context, as

$$
(B(u, v))_{n}=-\lambda^{n} u_{n-1} v_{n-1}+\lambda^{n+1} u_{n} v_{n+1}, \quad(A u)_{n}=\lambda^{2 n} u_{n} .
$$

The domain $D(A)$ is a dense subset of $H$. $A$ is a positive definite operator with eigenvalues

$$
0 \leqslant \lambda^{2} \leqslant \lambda^{4} \leqslant \lambda^{6} \leqslant \ldots
$$

We define the functional space $H^{s}=A^{-s / 2} H$ with the following scalar product and norm:

$$
(u, v)_{s}:=\sum_{n=1}^{\infty} \lambda^{2 n s} u_{n} v_{n}, \quad|u|_{s}:=\sqrt{(u, u)_{s}} .
$$

The dissipation mechanism provided by the singularity of the inviscid dyadic model comes from the fact that solutions of these equations fail to be in $H^{5 / 6}$ after the blow-up, so that the energy balance is no longer valid:

$$
\frac{1}{2} \frac{\mathrm{d}}{\mathrm{d} t}|u|^{2}-(f, u)=(B(u, u), u) \neq 0
$$

This lack of balance is caused by the blow-up solution, whose spectral scaling is $\left\langle\left|u_{k}\right|\right\rangle \sim k^{-1 / 3}$. Solutions with positive viscosity are always regular, which implies that they belong to $H^{s}$ for every $s \in \mathbb{R}$ and satisfies the energy balance. Nonetheless, the viscous regularization is mostly concentrated on high wavenumbers, and this is the reason we still observe in our simulations a $k^{-1 / 3}$ inertial range, which is reminiscent of the singularity produced by inviscid solutions; see [3-5]. 
The inviscid Voigt-dyadic model, on the other hand, does not produce any singularity when $\alpha>0$. This means that if initial data are in $H^{s}$, it will remain in $H^{s}$. This is proved in the appendix of this work. It implies that if the initial data have exponentially decaying modes, i.e. are smooth, then the global attractor keeps this exponentially decaying feature. This can be proved by following the arguments of [21].

As we will see in this section, this has consequences on the decaying behavior of the spectra of the viscous $\alpha$-dyadic model: the inertial range is shortened, which implies that fewer degrees of freedom are necessary to reproduce the large-scale features accurately. Since the large-scale statistics is unchanged, this result suggests the use of the Voigt regularization as a sub-grid model.

\subsection{Dimensional analysis}

Now, we follow [13] and [15] to investigate the energy distribution scale by scale for the 3D NSV equations. Let $\langle\cdot\rangle$ denote average with respect to an invariant measure for the NSV semi-group (such a measure is known to exist for the NSV, see [40]). For the NS equations, assuming that there exists an extensive range of wavenumbers, where the viscous dissipation does not play a significant role, one can show that the energy simply cascades through these length scales, with the rate being equal to the mean energy dissipation rate, $\epsilon=v\langle|\nabla \mathbf{u}|\rangle$. In [28], we prove that a similar scenario holds for the $\alpha$-energy, defined in (7), of the NSV equations. The same could be done here for the Voigt-dyadic model.

In [28], the authors argued via dimensional analysis to find the proper scaling for the inertial range. The extra parameter $\alpha$ generates several possible timescales, which further complicates the dimensional analysis. The correct scaling could only be settled by the employment of numerical simulations. In this section, we argue in the same lines as in [28], but due to different results for our numerical simulations we have to argue for a different timescale. This shows the limitations of dimensional analysis arguments. Let $\mathbf{u}$ be a solution of the NSV equations. We denote by $\epsilon_{\alpha}$ the mean $\alpha$-energy dissipation rate,

$$
\epsilon_{\alpha}=v\left\langle|\nabla \mathbf{u}|^{2}\right\rangle .
$$

Now, we want to investigate the distribution of the inviscid conserved quantity, the $\alpha$-energy, scale by scale. We define the following characteristic velocities at scale $k$ :

$$
U_{k}^{(0)}:=\left\langle\left|\mathbf{u}_{k}\right|^{2}\right\rangle^{1 / 2}
$$

and

$$
U_{k}^{(\alpha)}:=\left(1+\alpha^{2} k^{2}\right)\left\langle\left|\mathbf{u}_{k}\right|^{2}\right\rangle^{1 / 2} .
$$

We denote the characteristic $\alpha$-energy at scale $k$ by

$$
S_{2}^{\alpha}(k):=\frac{1}{2} \mathbf{U}_{k}^{(0)} \mathbf{U}_{k}^{(\alpha)}=\frac{1}{2}\left(1+\alpha^{2} k^{2}\right)\left(\mathbf{U}_{k}^{(0)}\right)^{2} .
$$

The characteristic kinetic energy at scale $k$ by

$$
S_{2}(k):=\frac{1}{2}\left(\mathbf{U}_{k}^{(0)}\right)^{2} .
$$

With this notation, we can write the $\alpha$-energy as

$$
S_{2}^{\alpha}:=\sum_{k} S_{2}^{\alpha}(k)=\frac{1}{2} \sum_{k} \mathbf{U}_{k}^{(0)} \mathbf{U}_{k}^{(\alpha)}
$$

and the kinetic energy as

$$
S_{2}:=\sum_{k} S_{2}(k)=\frac{1}{2} \sum_{k}\left(\mathbf{U}_{k}^{(0)}\right)^{2} .
$$


In the inertial range, the $\alpha$-energy transfer timescale can be defined as

$$
t_{k}^{\text {transf }}=\frac{S_{2}^{\alpha}(k)}{\epsilon_{\alpha}} .
$$

Following the arguments used by Kraichnan in $[13,25,26]$ we may argue that in the inertial range, the eddies of size $k^{-1}$, in average, transfer their characteristic $\alpha$-energy to neighboring eddies in the time, $t_{k}^{\text {transf }}$, it takes to travel their own length, $k^{-1}$, i.e.

$$
t_{k}^{\text {transf }}=\frac{1}{k U_{k}},
$$

where $U_{k}$ is the characteristic velocity at scale $k$. Substituting (16) into (15), and setting $\alpha=0$, we recover the $k^{-2 / 3}$ scaling for the inertial range of the $S_{2}$ structure function, which was theoretically predicted for the NS equations by Kolmogorov in [24]. (We remark that the $k^{-2 / 3}$ scaling for the structure function is commonly quoted in terms of its correspondent energy spectrum density, which obeys the $k^{-5 / 3}$ power law.) This sort of argument also leads to the double cascading scenario for 2D turbulence described in [25].

For the NSV case, the situation is complicated due to the fact that we have two different characteristic velocities, $U_{k}^{(0)}$ and $U_{k}^{(\alpha)}$. In fact, any log-convex combinations of them would give us a possible characteristic velocity; see, e.g., $[1,2,6,28]$.

Real world turbulent flows, however, present anomalous scaling, i.e. structure functions deviate significantly from the Kolmogorov predictions in [24]; see, e.g., [16, 36, 41]. This anomalous behavior is present in some phenomenological models, such as the Sabra shell models introduced in [36]. For example, in [36], the scaling computed for the $S_{2}$ structure function was -0.72 , slightly deviating from the Kolmogorov $-2 / 3$ scaling. The nature of inertial range intermittency is a topic of current intense research in the turbulence community; see, e.g., [16] for more details.

In [28], simulations of the Sabra-NSV shell model clearly displayed two distinct power laws for $S_{2}^{\alpha}$. For large values of $\alpha$ compared to the Kolmogorov dissipation length scale, $\eta:=\left(v^{3} / \epsilon\right)^{1 / 4}$, a range with scaling slightly deviating from the $k^{-2 / 3}$ Kolmogorov scaling (see section 4 for more details) and a range with a nearly power zero scaling were observed; see figure 1 . This distribution was explained by setting up the transfer timescale $t_{k}^{\text {transf }}$ in equation (16), derived from the translational velocity, $U_{k} \sim U_{k}^{(0)}$ :

$$
t_{k}^{\text {transf }}=\frac{1}{k U_{k}^{(0)}} \sim \frac{\left(1+\alpha^{2} k^{2}\right)^{1 / 2}}{k\left(U_{k}^{(0)}\right)^{1 / 2}\left(U_{k}^{(\alpha)}\right)^{1 / 2}} .
$$

In fact, substituting the expression above into (15), we obtain

$$
S_{2}^{\alpha}(k) \sim \epsilon^{2 / 3} k^{-2 / 3}\left(1+\alpha^{2} k^{2}\right)^{1 / 3}
$$

Therefore, for $k \ll \alpha^{-1}$, we have a $k^{-2 / 3}$ range, while for $\alpha \approx k^{-1}$ we have a power zero range, just as is observed in the shell model simulations in [28]. From this relation, we also obtain the energy transfer timescale for the Voigt-Sabra model:

$$
t_{k}^{\text {sabra }} \sim \epsilon_{\alpha}^{-1 / 3}\left(1+\alpha^{2} k^{2}\right)^{1 / 3} k^{-2 / 3} .
$$

This scenario of two power laws in the inertial range was first proposed in [14] for the NS- $\alpha$ model, and then for other $\alpha$ models in [1, 2, 6, 20, 31, 34, 28].

The numerical experiments of the Voigt regularization of the singular dyadic shell model reported in this section show that despite the similar scaling structure of the equations, our results imply a different timescale. These simulations show a clean $k^{-2 / 3}$ scaling for the $S_{2}$ structure function without any intermittent corrections. As a matter of fact, all the observed $S_{n}$ 
structure functions displayed a clean $-n / 3$. This fact can only be explained via the Kraichnanlike arguments mentioned above by setting up the characteristic velocity as

$$
U_{k} \sim \frac{U_{k}^{(0)}}{1+\alpha^{2} k^{2}} .
$$

Indeed, substituting (20) into (16), and equating the result with (15) yields the observed scaling $\left(U_{k}^{(0)}\right)^{n} \sim \epsilon^{n / 3} k^{-n / 3}$.

Therefore, the energy transfer timescale for the Voigt-dyadic model is

$$
t_{k}^{\text {dyadic }} \sim \epsilon_{\alpha}^{-1 / 3}\left(1+\alpha^{2} k^{2}\right) k^{-2 / 3} .
$$

If we now compare (19) and (21), we see that while for the Voigt-Sabra model the transfer timescale approaches $\mathrm{O}(1)$ for large $k$ in the inertial range, the transfer timescale for the Voigtdyadic model grows to $\mathrm{O}\left(k^{4 / 3}\right)$. This explains the energy equidistribution range observed in the Voigt-Sabra simulations, and the reduced inertial range for the Voigt-dyadic model.

Therefore, by applying the Kraichnan-type analysis to the observed simulations, we observe that the different structures of the nonlinear terms of each of the shell models yield different transfer timescales, and ultimately, different spectra. Hence, the true spectra of the full NSV equations remain an open question, which can be answered only by direct numerical simulations which we intend to report in a follow-up work.

\subsection{Numerical experiments}

In this section, we discuss the results of numerical simulations of the Voigt-dyadic model.

The simulations were performed using the MATLAB stiff ODE solver ode15s. We found that letting MATLAB approximate the Jacobian of the system via finite differences was much faster than passing the actual Jacobian as a parameter to the solver. The use of an approximated Jacobian has no relevant effect on the quality of the numerical solutions, since the solver algorithm keeps the error under a predefined tolerance in any case.

Let us first define the $S_{n}$ structure function as

$$
S_{n}(k)=\left\langle\left|U_{k}(t)\right|^{n}\right\rangle, \quad k=1, \ldots, M,
$$

where $\langle\cdot\rangle$ means time average and $M$ is the number of modes used in the simulation. We denote by $k_{d}$ the dissipative cut-off wavenumber, where the inertial range ends and the dissipative range begins. Also, for a fixed force and fixed viscosity $\nu$, we denote by $\alpha_{c}$ the dissipative critical length scale such that for all values of $\alpha \geqslant \alpha_{c}$, the inertial range starts to shorten, and such that for all values of $\alpha<\alpha_{c}$, the inertial range (or, equivalently, the value of $k_{d}$ ) is unchanged. Finally, we define the value $T_{r}=\alpha_{c}^{2} / v$, which has a dimension of time, as the critical relaxation time of a given simulation.

The free parameters of the simulations are the viscosity $v$, which ranges from $10^{-12}$ to $10^{-6}$, the parameter $\alpha$, which ranges from $10^{-4}$ to $10^{5}$, and the forcing term $f$. We have tested several forcing schemes, varying from constant to highly oscillatory in time. We have also varied the spectral support of the forces, from being located only on the first few modes to forces applied in all wavenumbers. All simulations displayed the same spectral behavior as those discussed below, differing only at the total energy level, and thus we list only a few of them. All data and programs can be obtained upon request via the authors' e-mail addresses.

In order to keep the consistency of the results across all runs, the maximum wavenumber $M$ and the final time $T_{f}$ were fixed for a given force scheme. In all simulations, the final time 
Table 1. In this table, $\alpha_{c}$ is the critical length parameter such that for any $\alpha \geqslant \alpha_{c}$, the inertial range is shortened. Note the universality of the critical relaxation time parameter $T_{r}$. For all simulations, the forcing method was fixed: a mix of constant and highly oscillatory forcing modes in the first few wavenumbers. Final time $=T_{f}=2 \times 10^{7}$ in all runs.

\begin{tabular}{llll}
\hline$v$ & $\alpha_{c}$ & $T_{r}$ & $\alpha_{d}$ \\
\hline $1 \times 10^{-12}$ & $1.258925 \times 10^{-3}$ & $1.584893 \times 10^{6}$ & $6.309573 \times 10^{2}$ \\
$1 \times 10^{-11}$ & $3.981072 \times 10^{-3}$ & $1.584893 \times 10^{6}$ & $6.309573 \times 10^{2}$ \\
$1 \times 10^{-10}$ & $1.258925 \times 10^{-2}$ & $1.584893 \times 10^{6}$ & $6.309573 \times 10^{2}$ \\
$1 \times 10^{-9}$ & $3.981072 \times 10^{-2}$ & $1.584893 \times 10^{6}$ & $6.309573 \times 10^{2}$ \\
$1 \times 10^{-8}$ & $1.258925 \times 10^{-1}$ & $1.584893 \times 10^{6}$ & $6.309573 \times 10^{2}$ \\
$1 \times 10^{-7}$ & $3.981072 \times 10^{-1}$ & $1.584893 \times 10^{6}$ & $6.309573 \times 10^{2}$ \\
$1 \times 10^{-6}$ & $1.258925 \times 10^{0}$ & $1.584893 \times 10^{6}$ & $6.309573 \times 10^{2}$ \\
\hline
\end{tabular}

Table 2. Similar to table 1. Here we just show data for a different forcing scheme. Forcing = constant in all modes. Final time $=T_{f}=1 \times 10^{8}$ in all runs.

\begin{tabular}{llll}
\hline$v$ & $\alpha_{c}$ & $T_{r}$ & $\alpha_{d}$ \\
\hline $1 \times 10^{-12}$ & $1.584893 \times 10^{-3}$ & $2.511886 \times 10^{6}$ & $3.162278 \times 10^{3}$ \\
$1 \times 10^{-11}$ & $5.011872 \times 10^{-3}$ & $2.511886 \times 10^{6}$ & $3.162278 \times 10^{3}$ \\
$1 \times 10^{-10}$ & $1.584893 \times 10^{-2}$ & $2.511886 \times 10^{6}$ & $3.162278 \times 10^{3}$ \\
$1 \times 10^{-9}$ & $5.011872 \times 10^{-2}$ & $2.511886 \times 10^{6}$ & $3.162278 \times 10^{3}$ \\
$1 \times 10^{-8}$ & $1.584893 \times 10^{-1}$ & $2.511886 \times 10^{6}$ & $3.162278 \times 10^{3}$ \\
$1 \times 10^{-7}$ & $5.011872 \times 10^{-1}$ & $2.511886 \times 10^{6}$ & $3.162278 \times 10^{3}$ \\
$1 \times 10^{-6}$ & $1.584893 \times 10^{0}$ & $2.511886 \times 10^{6}$ & $3.162278 \times 10^{3}$ \\
\hline
\end{tabular}

was chosen in order to satisfy the following convergence criterion: if at the final time $T_{f}>T_{r}$ the solution $U$ satisfied

$$
\max _{k \leqslant M}\left\{\frac{\left\langle\left|U_{k}^{2}(\cdot)-\left\langle\left|U_{k}\right|^{2}\right\rangle_{I}\right|^{2}\right\rangle_{I}^{1 / 2}}{\left\langle\left|U_{k}\right|^{2}\right\rangle_{I}}\right\}<10^{-3}, \quad I=\left[T_{f}-T_{r}, T_{f}\right],
$$

then the numerical experiment converged. The reason for measuring the convergence in a time window $I=\left[T_{f}-T_{r}, T_{f}\right]$ of length $T_{r}$ is that the critical relaxation time is the most relevant time unit in this problem. Moreover, we shall see that once the solutions converge, the value of $T_{r}$ depends on the forcing term $f$ only. In practice, we choose $T_{f}>10 \times T_{r}$ and verify that $T_{f}$ satisfies the convergence criteria a posteriori.

The values of $S_{n}, T_{r}, k_{d}$ and $\alpha_{c}$ were observed from the numerical results. In order to standardize the simulations, an objective way of measuring $k_{d}$ was deemed necessary. As such, we have stipulated $k_{d}$ as the smaller wavenumber $k$ such that $S_{2}(k+1) / S_{2}(k) \leqslant 10^{-1 / 2}$, if $k \geqslant k_{d}$. In our experience, this corresponds well with the eyeballed notion of $k_{d}$ when one sees the graph of $S_{2}$ versus $k$ in a semi-log plot; otherwise, it was an arbitrary decision with no deeper reason behind it. The coefficient $\alpha_{c}$ was defined as the smaller $\alpha$ that makes the value of $k_{d}$ drop.

In figure 2, we display the semi-log plots of the scaled structure functions, $S_{n}(k) \cdot k^{n / 3}$, for several values of $\alpha$ in each plot. First, observe that there is no intermittent scaling for any structure function, i.e. $S_{n}(k) \sim k^{-n / 3}$ in the inertial range with no deviations. We also observe that the power-law range is reduced as the parameter $\alpha$ is increased.

Tables 1 and 2 show that, indeed, the relevant parameter is the critical relaxation time. We observe that $\alpha_{c}$ changes with $v$ in such a way that $T_{r}$ is a constant, which is mainly force 

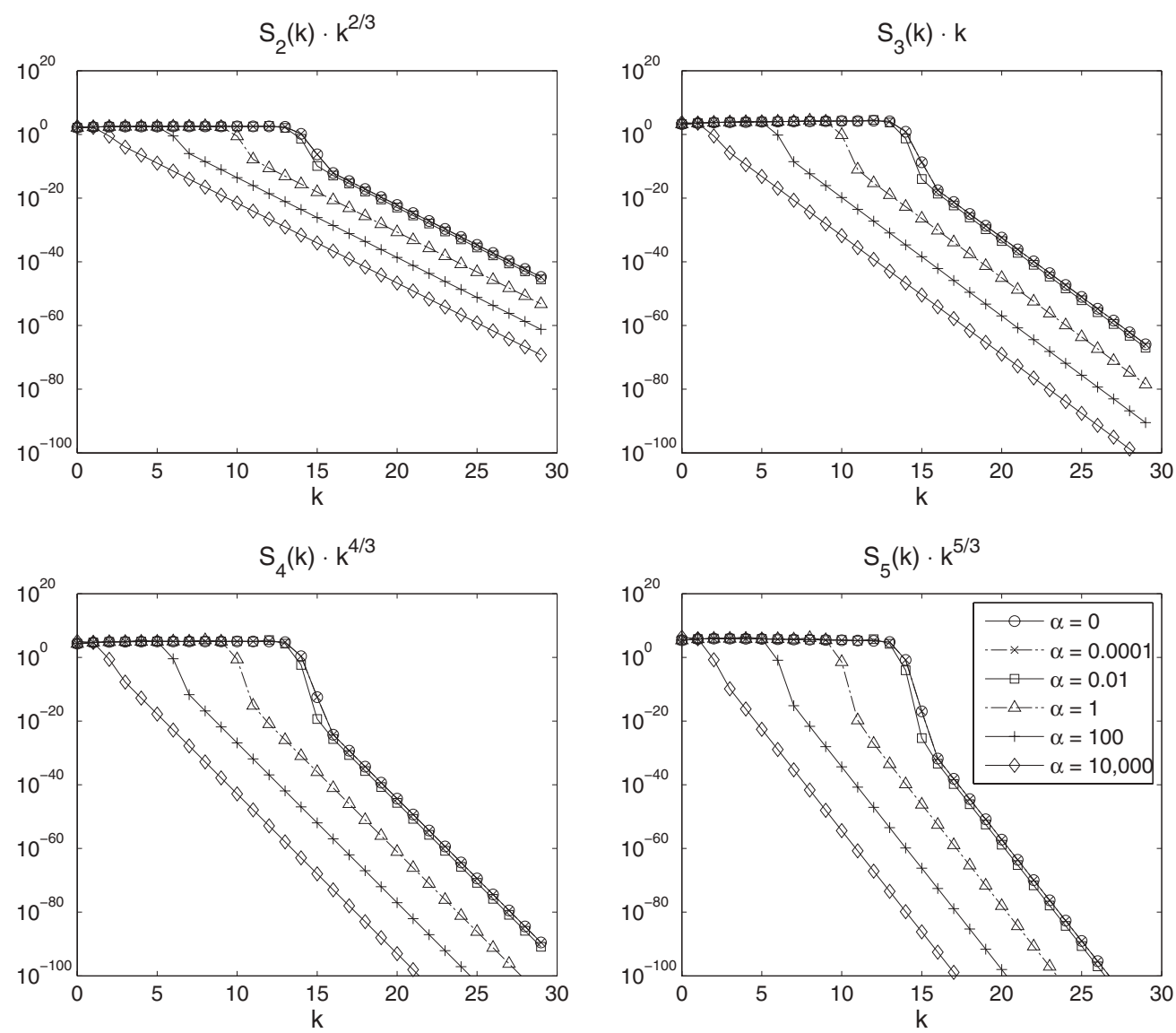

Figure 2. Semi-log plot of compensated structure functions, $S_{n}(k) \cdot k^{n / 3}$. Viscosity $=v=10^{-12}$ and several values of $\alpha$. Forcing: constant in time $=f_{k}$ for each mode $k$. Final time $=T_{f}=1 \times 10^{8}$ in all runs.

dependent. Moreover, for every $v$ we have $\alpha_{c}(v) \sim T_{r} v^{1 / 2}$. This law is illustrated in figure 3 . This is very useful, since for each fixed force, one can run a cheap simulation with large viscosity, and from that information, one can obtain the critical relaxation time $T_{r}$. With this coefficient in hand, for every fixed viscosity, it is possible to tune up the parameter $\alpha$ above the critical $\alpha_{c}$ so that the degrees of freedom are reduced. This can be key information for the use of the Voigt regularization as a sub-grid model.

We denote by $\alpha_{d}$ the critical value of the parameter $\alpha$ such that for all values of $\alpha \geqslant \alpha_{d}$ the inertial range disappears, giving way to a unique dissipative range. As can be observed in the tables, this critical value is force dependent, but, surprisingly, it is viscosity independent. This shows the power of the Voigt regularization, and so far we have not obtained any explanation for this behavior, which is certainly related to the regularity result proved in the appendix.

In figure 4 , we show the graph of $S_{2}$ with varying $v$, for fixed $\alpha$ and fixed force. As expected, one observes that $k_{d}$ decays with increasing $v$. This decay seems to be regular. Indeed, by fitting the data we were able to obtain the law $k_{d}(v) \sim-\log (\nu)+B_{f}$ for $\alpha_{c} \leqslant \alpha \leqslant \alpha_{d}$, where $B_{f}$ is a constant dependent only on the force. 


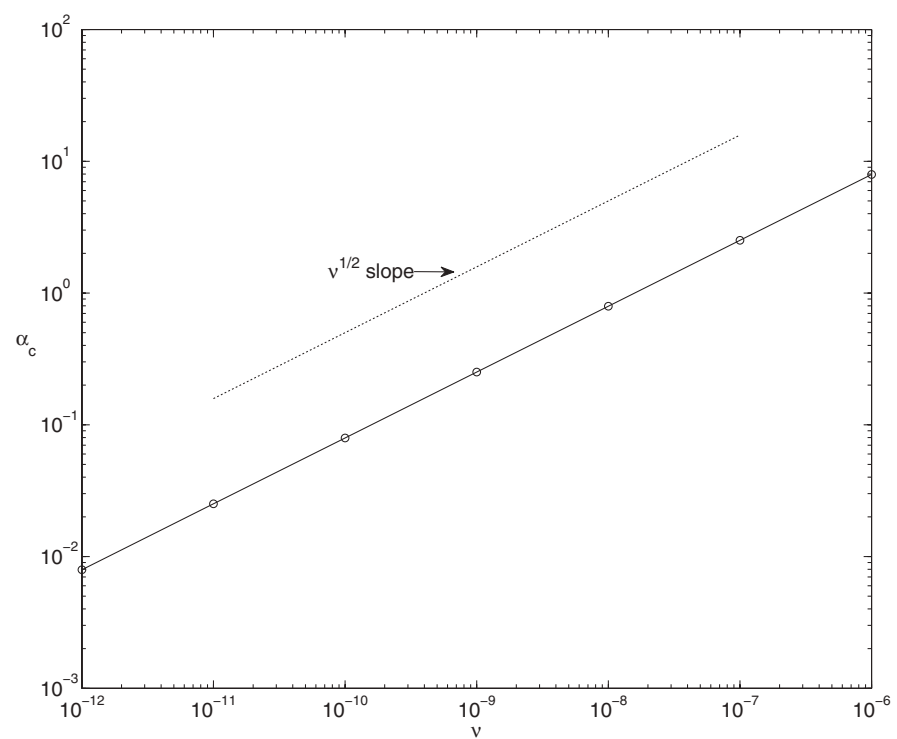

Figure 3. Relationship between the critical parameter $\alpha_{c}$ and viscosity $v$ : semi-log plot of $\alpha_{c}(v) \sim T_{r} v^{1 / 2}$, where the critical relaxation time $T_{r}$ is only force dependent. Viscosity $=v=10^{-12}$ and several values of $\alpha$. Forcing: constant in time $=f_{k}$ for each mode $k$. Final time $=T_{f}=1 \times 10^{8}$ in all runs.

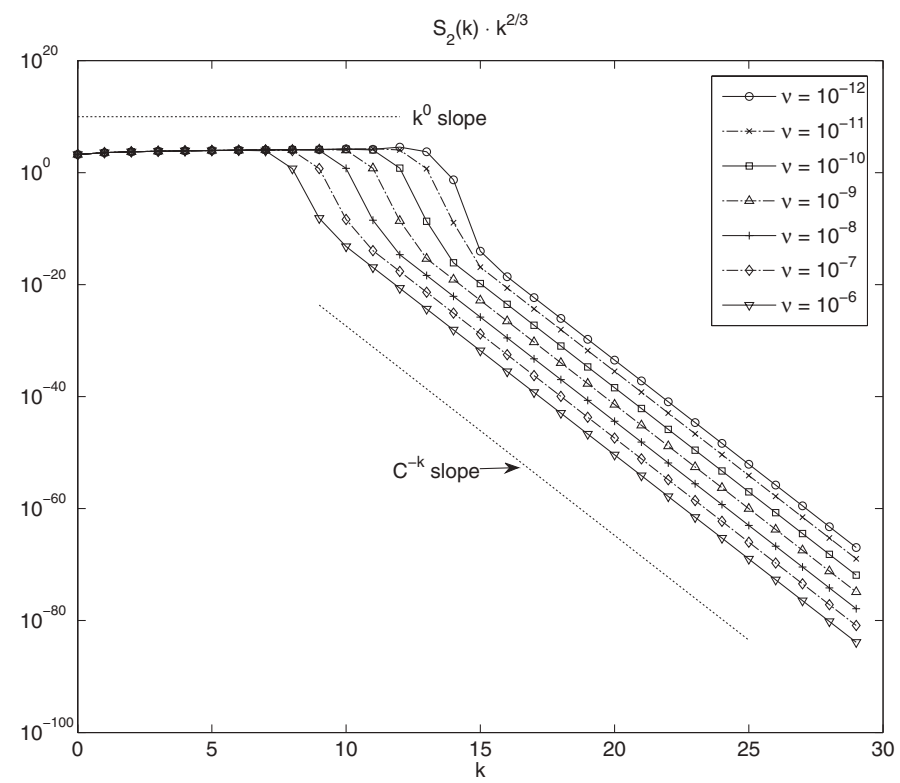

Figure 4. Semi-log plot of the compensated $S_{2}$ structure function, $S_{2}(k) \cdot k^{2 / 3} \cdot \alpha=0.01$, and several values of the viscosity $v$. Forcing: constant in time $=f_{k}$ for each mode $k$. Final time $=T_{f}=$ $1 \times 10^{8}$ in all runs. Here, $C \approx 10^{3.74}$. 


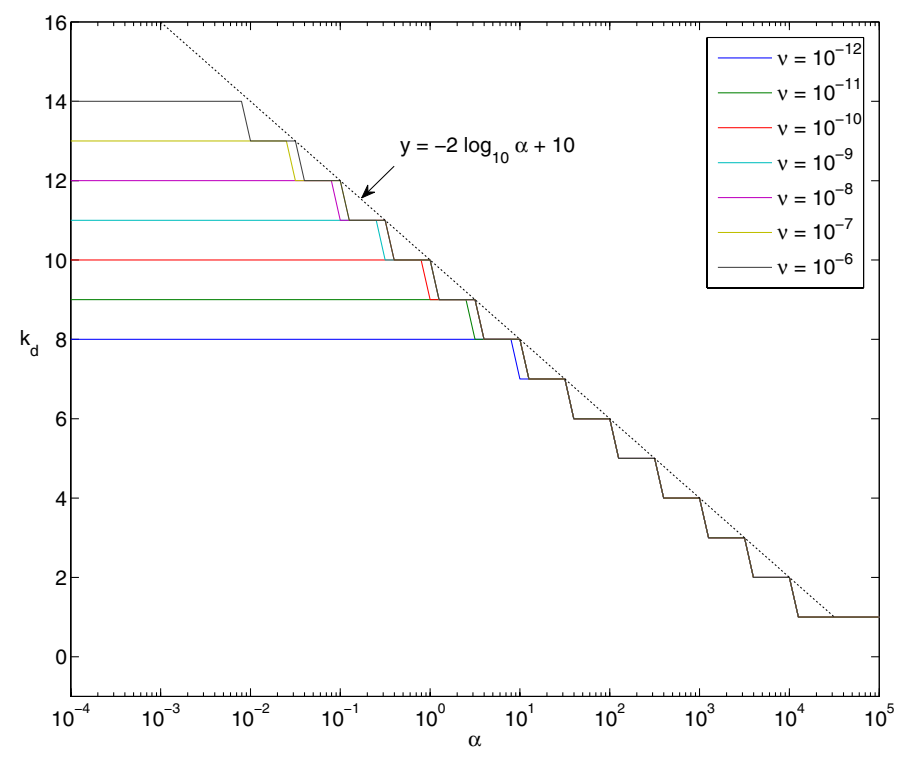

Figure 5. Dissipative cut-off wavenumber $\left(k_{d}\right)$ as a function of $\alpha$ for several values of viscosity $\nu$. Forcing: constant in time $=f_{k}$ for each mode $k$. Final time $=T_{f}=1 \times 10^{8}$ in all runs.

Figure 5 shows the values of $k_{d}$ as a function of $\alpha$ for selected values of $v$. The curves corresponding to different $v$ overlap as $\alpha$ grows, being practically indistinguishable from each other for $\alpha>10$. This figure makes it evident that, for any $v$ and small $\alpha, k_{d}$ assumes a constant value up to a critical value $\alpha_{c}$ (such a value depends on $v$ ). After that, $k_{d}$ decreases in regular steps (the curve $y(\alpha)=-2 \log _{10} \alpha+10$ fits the edges of the steps very well) until $\alpha$ assumes the dissipative critical value $\alpha_{d}$. After that, for $\alpha>\alpha_{d}, k_{d}$ is again a constant, this time with the minimum possible value 1 .

This behavior is also observed when we choose different forcing terms. In general, the steps of $k_{d}$ fit a line $y(\alpha)=-2 \log _{10} \alpha+c_{f}$, where $c_{f}$ is a constant that depends on the force $f$ only. Once again, this is very useful information for the aim of sub-grid modeling, because if such a law is true, for any given $v$ and $k$, one is able to choose $\alpha$ such that the dissipative cut-off wavenumber is exactly $k$. In the case of figure 5 , it is sufficient to choose $\alpha$ to the left of the line, that is, $\alpha>10^{\frac{10-k}{2}}$. In the general case, $\alpha$ must satisfy the condition $\alpha>10^{\frac{c_{f}-k}{2}}$. The value of $c_{f}$ can be obtained from $\alpha_{d}$, via linear interpolation: since $k_{d}$ jumps from 2 to 1 at $\alpha_{d}$, one has

$$
-2 \log _{10} \alpha_{d}+c_{f}=2 \quad \therefore c_{f}=2+2 \log _{10} \alpha_{d} .
$$

\section{Conclusions}

In this work, we have investigated the spectral scaling of the Navier-Stokes-Voigt model as the relaxation time parameter $\alpha^{2} / v$ varies. We found that the inertial range is shortened as $\alpha$ is increased above a critical length scale $\alpha_{c}$ such that the relaxation time satisfies $\alpha^{2} / \nu \geqslant T_{r}=\alpha_{c}^{2} / \nu$, a critical relaxation time which is only force dependent. Indeed, we have numerically obtained a more precise law describing the dissipative cut-off, $k_{\nu, f}$, for a fixed viscosity $v$ and force $f$ : the law reads $k_{v, f}(\alpha)=-2 \log \alpha+c_{f}$, where $c_{f}$ is a forcedependent constant. 
The statistics of scales larger than this critical length were insensitive to the regularization. This means that the effects of the Voigt regularization were constrained to the small scales, where dissipation is more prominent, and timescales tend to be very short, which usually increases computational costs. This is consistent with the numerical simulations of the twodimensional Navier-Stokes equations and MHD flows reported in [27], where the Voigt regularization was shown to improve resolution in coarse meshes.

We would also like to point out some very interesting results concerning the sub-grid effects of $\alpha$ regularization of three-dimensional flows obtained in [18, 19]. It is our intention to compare the three-dimensional simulations of the Voigt model with those observed in $[18,19]$ in a future work.

Our results are compatible with the energy cascade explanation for the phenomena of drag reduction in viscoelastic flows. The elastic contribution increases the energy transfer timescales, which follows (21). This faster energy transfer implies that the viscous term becomes more dominant in large scales, so that small-scale kinematics, which is dominant close to the boundary, gets less excited. This consequently shortens the inertial range and reduces the drag.

We recall that the results obtained in this work are very different from those observed in [28], where the Voigt model was studied via Sabra shell model simulations. There, simulations showed two power-law scenarios, commonly observed in the simulations of $\alpha$-regularization models. This shows the limitations of dimensional analysis arguments for studying spectral scaling of large coupled systems of equations arising in fluid mechanics without looking at the details of the coupling terms. This disparity in the results is the main motivation for the use of extensive three-dimensional numerical simulations in a coming work to settle down the correct scaling and argue for the use of the Voigt regularization as a sub-grid model.

We conjecture that the reduced degrees of freedom are mainly due to the regularization effect proved in the appendix. We believe that a large class of viscoelastic modifications of the Navier-Stokes equations may be useful as a large-eddy simulation tool as long as the elasticity effects are concentrated in small scales, and as long as it does not require extra boundary conditions.

\section{Acknowledgments}

RB was supported by the CAPES Foundation, Ministry of Education of Brazil. FR was supported by a CNPq research fellowship (grant no. 305309/2010-2). FR also wants to thank Edriss S Titi for fruitful discussions, and the hospitality of the Department of Mathematics of the University of California at Irvine, where part of this work was completed during a visit supported by the CNPq Cooperation Project 490124/2009-7. The authors would like to thank the referees' valuable comments that greatly improved this manuscript.

\section{Appendix. Regularity of the Voigt-dyadic model}

The inviscid Voigt-dyadic model is the system of equations

$$
\left(1+\alpha^{2} \lambda^{2 n}\right) \frac{\mathrm{d} u_{n}}{\mathrm{~d} t}-\lambda^{n} u_{n-1}^{2}+\lambda^{n+1} u_{n} u_{n+1}=f_{n},
$$

for $n=1,2,3, \ldots$, with the boundary condition $u_{0}=0$, and where $\lambda>1$ is the shell spacing parameter. The parameter $\alpha \geqslant 0$ plays the same role as described in (3). 
Theorem 1. Let $u^{0} \in H^{s}, f \in L^{\infty}\left([0, T], H^{s}\right)$, then for every $\alpha>0$ and $T>0$, there exists a unique solution $u(t)=\left\{u_{n}(t)\right\}_{n \in \mathbb{Z}} \in C\left([0, T], H^{s}\right)$ of equation (A.1) with the initial condition $u(0)=u^{0}$.

Proof. Let us begin with an inequality for the nonlinear term $B(u, v)$, defined in (12), which will be very important in this proof. Let $u, w \in H^{s}, s \in \mathbb{Z}$ and $v \in H^{1}$, then

$$
\begin{aligned}
\left|(B(u, v), w)_{s}\right|= & \left|\sum_{n} \lambda^{n} \lambda^{2 s n} u_{n-1} v_{n-1} w_{n}+\sum_{n} \lambda^{n+1} \lambda^{2 s n} u_{n} v_{n+1} w_{n}\right| \\
\leqslant & \lambda^{s+1}\left|\sum_{n}\left[\left(\lambda^{s(n-1)} u_{n-1}\right)\left(\lambda^{n-1} v_{n-1}\right)\right]\left(\lambda^{s n} w_{n}\right)\right| \\
& +\left|\sum_{n}\left[\left(\lambda^{s n} u_{n}\right)\left(\lambda^{n+1} v_{n+1}\right)\right]\left(\lambda^{s n} w_{n}\right)\right| \\
\leqslant & \lambda^{s+1}\left(\sum_{n} \lambda^{2 s n}\left|w_{n}\right|^{2}\right)^{1 / 2}\left(\sum_{n}\left(\lambda^{2 s(n-1)}\left|u_{n-1}\right|^{2}\right)\left(\lambda^{2(n-1)}\left|v_{n-1}\right|^{2}\right)\right)^{1 / 2} \\
& +\left(\sum_{n} \lambda^{2 s n}\left|w_{n}\right|^{2}\right)^{1 / 2}\left(\sum_{n}\left(\lambda^{2 s n}\left|u_{n}\right|^{2}\right)\left(\lambda^{2(n+1)}\left|v_{n+1}\right|^{2}\right)\right)^{1 / 2} \\
\leqslant & C_{\lambda}\|w\|_{s}\left(\sup _{n}\left(\lambda^{2 n}\left|v_{n}\right|^{2}\right) \sum_{n} \lambda^{2 s n}\left|u_{n}\right|^{2}\right)^{1 / 2} \\
\leqslant & C_{\lambda}\|u\|_{s}\|v\|_{1}\|w\|_{s} .
\end{aligned}
$$

This proves that the operator $B(u, v)$ can be extended such that $B: H^{s} \times H^{1} \rightarrow H^{s}$, and that for $u \in H^{s}, v \in H^{1}$, one has

$$
\|B(u, v)\|_{s} \leqslant C_{\lambda}\|u\|_{s}\|v\|_{1} .
$$

It is easy to see that the roles of $u$ and $v$ can be interchanged in inequality (A.2), and, therefore, for $u \in H^{s}, v \in H^{1}$, one also has

$$
\|B(v, u)\|_{s} \leqslant C_{\lambda}\|u\|_{s}\|v\|_{1} .
$$

We now continue with the proof of the theorem. It follows the arguments from [2] and [29], and, therefore, we will only give a brief sketch of how it works in the dyadic model case. Let us first rewrite equation (A.1) as

$$
\begin{aligned}
\frac{\mathrm{d} v_{n}}{\mathrm{~d} t} & =N(v):=-v \lambda^{2 n} u_{n}+\lambda^{n} u_{n-1}^{2}-\lambda^{n+1} u_{n} u_{n+1}+f_{n}, \\
v_{n} & =\left(1+\alpha^{2} \lambda^{2 n}\right) u_{n}
\end{aligned}
$$

We start by proving that if $v^{0}=\left(1+\alpha^{2} A\right) u^{0} \in H^{-1}\left(u^{0} \in H^{1}\right)$, then there exists a short time $T_{*}\left(\left\|v^{0}\right\|_{-1}\right)$ such that equation (A.5) has a unique solution in $C^{1}\left(\left[-T_{*}, T_{*}\right], H^{-1}\right)$. The proof follows from the Picard iteration scheme, once we prove that $N(v)$ is locally Lipschitz continuous in $H^{-1}$.

It follows from inequalities (A.3) and (A.4) and from Poincaré's inequality that for every $u^{(1)}, u^{(2)} \in H^{1} \subset H$, the bound

$$
\left\|B\left(u^{(1)}, u^{(2)}\right)\right\|_{-1} \leqslant C_{\lambda}\left\|u^{(1)}\right\|_{1}\left\|u^{(2)}\right\|_{1}
$$

holds. 
Now, if we let $v^{(i)}=\left(1+\alpha^{2} A\right) u^{(i)}, i=1,2$, we have from (A.6) the bilinearity of $B(\cdot, \cdot)$, the triangle inequality, and from the equivalence of the norms $\|u\|_{1}$ and $\|v\|_{-1}$ that

$$
\begin{aligned}
\left\|N\left(v^{(1)}\right)-N\left(v^{(2)}\right)\right\|_{-1} & \leqslant C\left\|u^{(1)}-u^{(2)}\right\|_{1}\left(\left\|u^{(1)}\right\|_{1}+\left\|u^{(2)}\right\|_{1}\right) \\
& \leqslant C R\left\|v^{(1)}-v^{(2)}\right\|_{-1},
\end{aligned}
$$

where $R$ is a constant such that $\left\|u^{(1)}\right\|_{1},\left\|u^{(2)}\right\|_{1} \leqslant R / 2$. This implies that $N(v)$ is locally Lipschitz in $H^{-1}$. Now, to prove that the solution remains bounded, note that if we denote by $\left[0, T_{\max }\right)$ the maximal interval of existence where $u \in C\left(\left[0, T_{\max }\right), H^{1}\right)$, then by taking the duality product in (A.5) with $u$, we have

$$
\frac{1}{2} \frac{\mathrm{d}}{\mathrm{d} t}\left(|u|^{2}+\alpha^{2}\|u\|_{1}^{2}\right) \leqslant\|f\|_{-1}|u| \leqslant \frac{\|f\|_{-1}}{\alpha}\left(|u|^{2}+\alpha^{2}\|u\|_{1}^{2}\right)^{1 / 2} .
$$

From this inequality, it follows that $u(t) \in C\left([0, \infty), H^{1}\right)$, if $u_{0} \in H^{1}$.

Now, to prove the general case, where $u_{0} \in H^{s}$, note that if, again, we let $v^{(i)}=$ $\left(1+\alpha^{2} A\right) u^{(i)}, i=1,2$, we have from inequalities (A.3) and (A.4) the bilinearity of $B(\cdot, \cdot)$, the triangle inequality, and from Poincaré's inequality that

$$
\begin{aligned}
& \left\|N\left(v^{(1)}\right)-N\left(v^{(2)}\right)\right\|_{s} \leqslant C\left\|u^{(1)}-u^{(2)}\right\|_{s}\left(\left\|u^{(1)}\right\|_{1}+\left\|u^{(2)}\right\|_{1}\right) \\
& \quad \leqslant C\left\|v^{(1)}-v^{(2)}\right\|_{s-2}\left(\left\|u^{(1)}\right\|_{1}+\left\|u^{(2)}\right\|_{1}\right) \leqslant C_{\lambda} R\left\|v^{(1)}-v^{(2)}\right\|_{s},
\end{aligned}
$$

where $R$ is a constant such that $\left\|u^{(1)}\right\|_{1},\left\|u^{(2)}\right\|_{1} \leqslant R$. This implies that $N(v)$ is locally Lipschitz in $H^{s}$, and, therefore, the Picard iteration scheme provides a local in time regular solution.

Now, to prove that the solution remains bounded, we proceed by induction. Suppose that for any $u^{0} \in H^{s}, s \in \mathbb{Z}$, we know that the solution of (A.5) remains bounded in $H^{r-1}, r \leqslant s$. We will show that this solution also remains bounded in $H^{r}$.

Indeed, denoting by $\left[0, T_{\max }^{(r)}\right)$ the maximal interval of existence, where $u \in$ $C\left(\left[0, T_{\max }^{(r)}\right), H^{r}\right)$, then by taking the duality product in (A.5) with $A^{r-1} u$, we obtain

$$
\begin{aligned}
\frac{1}{2} \frac{\mathrm{d}}{\mathrm{d} t}\left(\|u\|_{r-1}^{2}+\alpha^{2}\|u\|_{r}^{2}\right) & \leqslant\left|\left(A^{\frac{r-1}{2}} B(u, u), A^{\frac{r-1}{2}} u\right)\right|+\left|\left(A^{\frac{r-1}{2}} f, A^{\frac{r-1}{2}} u\right)\right| \\
& \leqslant C\|u\|_{r-1}^{2}\|u\|_{1}+\|f\|_{r-1}\|u\|_{r-1} .
\end{aligned}
$$

Since we have assumed that all norms of $u$ up to order $r-1$ were bounded, it follows easily from (A.10) that the $r$-norm of $u$ is bounded as well. We want to remark that all the preceding inequalities were formal, but they can all be made rigorous by truncating $u$ as $P_{M}(u):=\sum_{n=1}^{M} u_{n}$, so that all inequalities above hold for $P_{M}(u)$. Then, one may let $M \rightarrow \infty$ and use classical compactness theorems as described in $[8,44]$. This finishes the proof of the theorem.

Remark. It is easy to see from the proof above that we have indeed algebraic bounds in time. This is important if we want to control the effects of large oscillations in the statistical behavior of the solutions.

\section{References}

[1] Cao C, Holm D and Titi E S 2005 On the Clark- $\alpha$ model of turbulence: global regularity and long-time dynamics J. Turbul. 19 1-11

[2] Cao Y, Lunasin E M and Titi E S 2006 Global well-posedness of the three-dimensional viscous and inviscid simplified Bardina turbulence models Commun. Math. Sci. 4 823-84

[3] Cheskidov A 2008 Blow-up in finite time for the dyadic model of the Navier-Stokes equations Trans. Am. Math. Soc. 360 5101-20

[4] Cheskidov A, Friedlander A and Pavlović N 2010 An inviscid dyadic model of turbulence: the global attractor Discrete Contin. Dyn. Syst. 26 781-94 
[5] Cheskidov A, Friedlander S and Pavlović N 2007 Inviscid dyadic model of turbulence: the fixed point and Onsager's conjecture J. Math. Phys. 48065503

[6] Cheskidov A, Holm D, Olson E and Titi E S 2005 On a Leray- $\alpha$ model of turbulence Proc. R. Soc. Lond. A $461629-49$

[7] Chorin A J and Hald O H 2005 Viscosity-dependent inertial spectra of the Burgers and Korteweg-de VriesBurgers equations Proc. Natl Acad. Sci. USA 102 3921-3

[8] Constantin P and Foias C 1988 Navier-Stokes Equations (Chicago, IL: University of Chicago Press)

[9] Constantin P, Levant B and Titi ES 2006 Analytic study of shell models of turbulence Physica D $219120-41$

[10] Doi M and Edwards S F 1989 The Theory of Polymer Dynamics (Oxford: Oxford University Press)

[11] Constantin P, Weinan E and Titi E S 1994 Onsager's conjecture on the energy conservation for solutions of Euler's equation Commun. Math. Phys. $165207-9$

[12] Eyink GL and Sreenivasan K R 2006 Onsager and the theory of hydrodynamic turbulence Rev. Mod. Phys. 78 87-135

[13] Foias C 1995 What do the Navier-Stokes equations tell us about turbulence? Harmonic Analysis and Nonlinear Differential Equations (Riverside, CA: American Mathematical Society)

Foias C 1997 Contemp. Math. 208 151-80

[14] Foias C, Holm D and Titi ES 2001 The Navier-Stokes- $\alpha$ model of fluid turbulence Physica D 152-153 505-19

[15] Foias C, Manley O P, Rosa R and Temam R 2001 Navier-Stokes Equations and Turbulence (Encyclopedia of Mathematics and its Applications vol 83) (Cambridge: Cambridge University Press)

[16] Frisch U 1995 Turbulence. The Legacy of A N Kolmogorov (Cambridge: Cambridge University Press)

[17] Geurts B J, Kuczaj A and Titi E S 2008 Regularization modeling for large-eddy simulation of homogeneous isotropic decaying turbulence J. Phys. A: Math. Theor. 41344008

[18] Graham J P, Holm D, Mininni P D and Pouquet A 2011 The effect of subfilter-scale physics on regularization models J. Sci. Comput. 49 21-34

[19] Graham J P, Holm D, Mininni P D and Pouquet A 2007 Highly turbulent solutions of the Lagrangian-averaged Navier-Stokes $\alpha$ model and their large-eddy simulation potential Phys. Rev. E 76056310

[20] Ilyin A A, Lunasin EM and Titi E S 2006 A modified-Leray- $\alpha$ sub-grid scale model of turbulence Nonlinearity 19 879-97

[21] Kalantarov V and Titi E S 2009 Global attractors and determining modes for the 3D Navier-Stokes-Voight equations Chin. Ann. Math. Ser. B 30 697-714

[22] Kalantarov V, Levant B and Titi E S 2009 Gevrey regularity of the global attractor of the 3D Navier-StokesVoight Equations J. Nonlinear Sci. 19 133-52

[23] Katz N and Pavlović N 2005 Finite time blow-up for a dyadic model of the Euler equations Trans. Am. Math. Soc. 357 695-708

[24] Kolmogorov A N 1941 The local structure of turbulence in incompressible viscous fluid for very large Reynolds numbers Dokl. Akad. Nauk SSSR 30 301-5

[25] Kraichnan R H 1967 Inertial ranges in two dimensional turbulence Phys. Fluids $101417-23$

[26] Kurien S, Taylor M A and Matsumoto T 2004 Cascade time scales for energy and helicity in homogeneous isotropic turbulence Phys. Rev. E 69066313

[27] Kuberrya P, Larios A, Rebholza L G and Wilsona N E 2012 Numerical approximation of the Voigt regularization for incompressible Navier-Stokes and magnetohydrodynamic flows Comput. Math. Appl. 64 2647-62

[28] Levant B, Ramos F and Titi E S 2010 On the statistical properties of the 3D incompressible Navier-Stokes-Voigt model Commun. Math. Sci. 8 277-93

[29] Larios A and Titi E S 2010 On the higher-order global regularity of the inviscid Voigt-regularization of threedimensional hydrodynamic models Discrete Contin. Dyn. Syst. 14 603-27

[30] Lesieur M 2008 Turbulence in Fluids (Berlin: Springer)

[31] Lunasin E M, Kurien S, Taylor M and Titi E S 2007 A study of the Navier-Stokes- $\alpha$ model for two-dimensional turbulence J. Turbul. 8 1-21

[32] Ladyzhenskaya O A 2000 In memory of A P Oskolkov J. Math. Sci. 99 799-801

[33] Larson R G 1998 The Structure and Rheology of Complex Fluids (Topics in Chemical Engineering) (Oxford: Oxford University Press)

[34] Lunasin E, Kurien S and Titi E S 2008 Spectral scaling of the Leray- $\alpha$ model for two-dimensional turbulence J. Phys. A: Math. Theor. 41344014

[35] Lumley J L 1969 Drag reduction by additives Annu. Rev. Fluid Mech. 1 367-84

[36] L'vov V S, Podivilov E, Pomyalov A, Procaccia I and Vandembroucq D 1998 Improved shell model of turbulence Phys. Rev. E 58 1811-22 
[37] Oskolkov A P 1973 Uniqueness and global solvability for boundary-value problems for the equations of motion of aqueous solutions of polymers Zap. Nauchn. Sem. LOMI 38 98-136

[38] Oskolkov A P 1980 On the theory of the Voight fluids Zap. Nauchn. Sem. LOMI $96233-6$

[39] Pope S B 2000 Turbulent Flows (Cambridge: Cambridge University Press)

[40] Ramos F and Titi E S 2010 Invariant measures of the Navier-Stokes-Voigt model, and their Navier-Stokes limit Discrete Contin. Dyn. Syst. 28 375-403

[41] She Z S, Jackson E and Orzag S A 1991 Structure and dynamics of homogeneous turbulence: models and simulations Proc. R. Soc. Lond. A 434 101-24

[42] Groisman A and Steinberg A 2001 Efficient mixing at low Reynolds numbers using polymer additives Nature 410905

[43] Tabor M and de Gennes P G 1986 A cascade theory of drag reduction Europhys. Lett. 2 519-22

[44] Temam R 1984 Navier-Stokes Equations. Theory and Numerical Analysis (Studies in Mathematics and its Applications) 3rd edn (Amsterdam: North-Holland)

[45] Thompson W 1865 On the elasticity and viscosity of metals Proc. R. Soc. Lond. A $14289-97$

[46] Voigt W 1892 Ueber innere Reibung fester Körper, insbesondere der Metalle Ann. Phys., Lpz. 283 671-93

[47] White C and Mungal G 2008 Mechanics and prediction of turbulent drag reduction with polymer additives Annu. Rev. Fluid Mech. 40 235-56

[48] Wilkinson W L 1960 Non-Newtonian Fluids (New York: Pergamon) 